\title{
Free-electron lasers sources for scientific applications
}

\author{
M.-E. Couprie and J.-M. Ortéga* \\ LURE, Bâtiment 209 D, Université Paris Sud, 91405 Orsay Cedex, France
}

\begin{abstract}
The free-electron laser ("FEL") has proven to be an invaluable source of radiation in the UV to far infrared spectral ranges. It is based on the amplification of an optical wave by a high energy electron beam oscillating transversely to its propagation. In the last decade, several FELs have been designed and built as user dedicated facilities. Presently, seven infrared FELs, such as CLIO at Orsay (France), are running more than 1000 hours/year for users performing experiments in various scientific fields. In the UV spectral range, the Super-ACO (Orsay, France) FEL is promoting applications in various fields. The main applications are described and prospects are given.
\end{abstract}

Keywords. Lasers - infrared - ultraviolet - free-electron lasers.

\section{Overview}

The free-electron laser (FEL), operated for the first time in 1978 [1] is based on the amplification of an optical wave by a high-energy electron beam. After about 20 years of research, it appears that the FEL is mature in the spectral range of UV to infrared, although intensive research is continuing in the field of short wavelength and high power [2].

The gain medium of a FEL is a high-energy electron beam (several $\mathrm{MeV}$ or more) passing through a transverse magnetic field called "undulator". The undulator causes an electron oscillation perpendicular to its direction of propagation and, therefore, couples it with the optical field. Undulators are also commonly used on storage rings as powerful UV and X ray sources [3]. The relative degree of coherence of the light produced by these sources is due to the periodicity of the undulator providing a higher spectral brilliance than ordinary synchrotron radiation. In addition, under certain conditions, the interaction in the undulator of the emitted radiation with the electron beam leads to light amplification [2]. This effect, when operating within an optical cavity (Fig. 1), is the principle of the Free-Electron Laser ("FEL").

The laser radiation is emitted by the FEL at the "resonance wavelength" $\lambda_{R}$ :

$$
\lambda_{R}=\lambda_{\mathrm{o}} / 2 \gamma^{2} \cdot\left(1+K^{2} / 2\right)
$$

$\lambda_{\mathrm{o}}$ is the magnetic period of the undulator (a few $\mathrm{cm}$ in practice);

$E=\gamma \mathrm{mc}_{2}$ is the electron beam energy (Einstein's formula) giving numerically for the relativistic factor: $\gamma \cong 2 . E(\mathrm{MeV})$;

$K=\mathrm{e} B \lambda_{\mathrm{o}} / 2 \pi m c$ is the deflection parameter proportional to the magnetic field $B$ of the undulator ( $K=1$ to 3 typically).
Tuning of the laser wavelength is done continuously by varying either the undulator gap, which modifies the value of the $K$ parameter, the electron energy or a combination of the two.

Typical electron energies are:

$$
\begin{array}{ll}
-5-10 \mathrm{MeV} \quad \text { to produce wavelengths in } & \begin{array}{l}
\text { far infrared } \\
\text { mid-infrared }
\end{array} \\
-10-50 \mathrm{MeV} & \mathrm{UV}-\mathrm{VUV} \\
-500-1000 \mathrm{MeV} & \mathrm{X} \text { rays } \\
-10 \text { to } 20 \mathrm{GeV} ? &
\end{array}
$$

The length of an accelerator is about $1 \mathrm{~m} / 5 \mathrm{MeV}$, except for electrostatic machines which are longer, but limited to a few $\mathrm{MeV}$.

Indeed, the most striking characteristic of the FEL is that the gain medium can be tuned to any wavelength. The lasing range is limited, in principle, only by the energies that can be reached by the accelerator, while attaining sufficiently good beam properties. However, at short wavelength, it is very difficult to obtain a sufficiently high optical gain.

In the infrared spectral range, the combination of high achievable gains and broadband metal mirrors leads to continuously tuning FELs over a very wide spectral range (1 or 2 orders of magnitude in wavelength). In the UV \& VUV, the reflectivity achievable with mirrors with today technology decrease rapidly at wavelengths shorter than about $200 \mathrm{~nm}$. Therefore, the shortest lasing wavelength is presently about $200 \mathrm{~nm}$ [4] and nowadays 5 FELs are operating at $300 \mathrm{~nm}$ or below. Coherent emission in the VUV range has been obtained by harmonic generation of an external laser focused on the electron beam [5]. In the future, short wavelength harmonics of the FEL itself could constitute a usable source. Another solution proposed to reach the short wavelength range is the single pass FEL called "SASE" (Self Amplified Spontaneous Emission). However,

*Correspondence and reprints.

Received April 26, 2000; revised September 26, 2000; accepted October 3, 2000. 


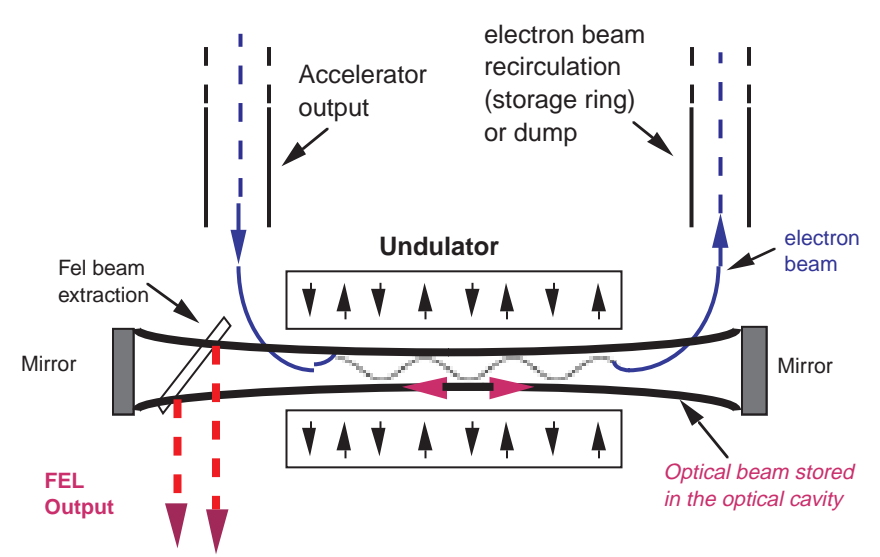

Figure 1. Scheme of an FEL in the oscillator configuration: A high energy electron beam passing through an undulator amplifies its own radiation stored in an optical cavity. The laser energy is extracted from the large electron kinetic energy.

this technique [6] requires very cumbersome machines and the shortest wavelength at which SASE has been observed is still in VUV [7]. Presently, efforts are made worldwide to promote the SASE technique and many studies are underway [11]. An alternative technique, X-ray and $\mathrm{MeV}$ production by FEL intracavity Compton backscattering, has been demonstrated [8]. The X-ray photon flux produced when an infrared FEL is backscattered by the same electrons that produce it, is still modest compared to the ones emitted by advanced synchrotron radiation machines; nevertheless, the use of the produced gamma-rays by Compton Back Scattering of the UV FEL is planned.

There is nevertheless a disadvantage in LINAC driven FELs: electron accelerator are cumbersome and expensive; the ionizing radiation that is produced by the electron losses require shielding and remote control. FELs using storage rings built as synchrotron radiation facilities have their cost somewhat reduced compared to the one of a dedicated FEL accelerator: this is the case of a few centers worldwide, which are capable of lasing in the UV range. The cost is much higher when a storage ring is built specially for FEL ( 2 such installations have been built and converted more or less into synchrotron radiation centers due to the lack of success of their FELs). Several projects of VUV storage ring FELs have been proposed [9]. An accelerator for infrared FEL is smaller than for shorter wavelengths, as only typically 5 to $50 \mathrm{MeV}$ are required. Typical infrared FELs (working on pulsed accelerator) are 10 to $20 \mathrm{~m}$ long. Recently very compact accelerator have been successfully tested [10]. Nevertheless, a shielded room for the accelerator is needed as well as a control room and several users rooms: the overall size of the installation does not change much. Far-infrared FELs using electrostatic accelerators have about the same size. The accelerator proposed for short wavelength SASE are rather huge $(10$ or $20 \mathrm{Gev})$ and more costly than a full synchrotron radiation center: the current

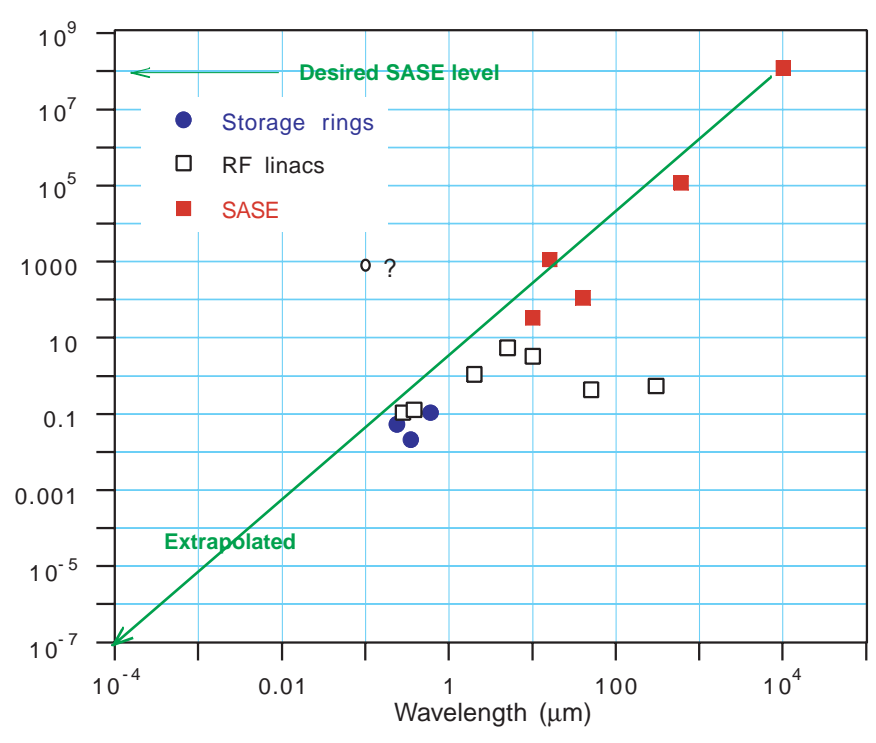

Figure 2. Optical gains (per pass in the undulator) measured with various FELs worldwide. Lasing requires, at least, a gain overwhelming the losses: mirror imperfect reflectivity, diffraction losses, beam extraction. A single pass device ("SASE") requires an amplification of approximately $10^{8}$.

projects would use accelerator built for particle physics at Stanford (SLAC) and Hamburg (DESY).

Soon after the invention of the FEL, projects of user facilities have been started. Due to technical difficulties, FEL facilities have been currently employed since only the late80s, most of them being fully operational since only a few years. Information can be found in the FEL conference proceedings [11] and on the web sites ${ }^{1}$.

\section{FEL coherence}

In general, FELs have a nearly diffraction limited transverse coherence. The output mode is generally close to a $\mathrm{TEM}_{00}$ gaussian mode.

The question of the longitudinal coherence is more complicated since most FELs utilise pulsed accelerators. Indeed, $\mathrm{cw}$ (electrostatic) accelerators are limited to low energy (a few $\mathrm{MeV}$ ) or become extremely cumbersome due to electrical insulation. Also, the optical gain being proportional to the peak electron current, it is easier to obtain sufficient values (several Amps at least), with pulsed devices. In typical pulsed radio-frequency linear accelerators ("RF linacs"), the electron pulse lengths are between 1 and $10 \mathrm{ps,} \mathrm{i.e.} 0.3$ to $3 \mathrm{~mm}$ long: such a pulse contains only a few optical wavelengths in the far-infrared range. If we assume that the laser

${ }^{1}$ See : http://sbfel3.ucsb.edu/www/vl_fel.html 
pulse length is equal to a given length $L$, and that the optical pulse train is fully coherent (transform limited), then its relative bandwidth is:

\section{$\Delta \lambda / \lambda=\alpha . \lambda / \mathrm{L} \quad$ where $\alpha \cong 0.4$ for a Gaussian shape.}

Therefore, the FEL bandwidth tends to become broad at long wavelengths. One could think that $L$ is equal to the electron pulse length. However, theory and experience of the FEL has shown that the relationship between the electron and light pulse length is more sophisticated and depends on many parameters such as the optical gain magnitude, the quality factor of the optical cavity and the slippage length (this quantity is the difference of path length along the undulator between electrons and light due to their different speeds). Consequently, the optical pulse can be either noticeably shorter or longer than the electron pulse length. The optical cavity length has much influence: the overlapp between the electron and photon pulses is adjusted by the cavity roundtrip time. At perfect synchronism, the laser cannot work, due to the slippage (which is called laser "lethargy"). Close to synchronism the optical pulse length is very short. At shorter lengths, the cavity acts like a dispersive element by lengthening the optical wavetrain. This effect is very useful in practice: the user can choose to work either in the short pulse mode, to perform time resolved experiment or non-linear studies, or in the small bandwith mode, to make, for example, non-linear spectroscopy. There is at least an order of magnitude of tuning range, depending on the FEL gain. When the slippage length is comparable with the bunch length, i.e. in mid-infrared $(10 \mu \mathrm{m})$ pulses as short as $200 \mathrm{fs}$ have been obtained [13], with $10 \%$ bandwidth. The smallest bandwidth ( $0.2 \%$ at CLIO, see below) seems to be limited by the accelerator stability as the wavelength decreases.

In far-infrared, with sufficient gain, the minimum bandwidth can be kept below $1 \%$. In the mm wavelength range an additional effect can be used to further reduce the bandwidth: the coherence effect between the successive laser pulses which yields a laser line composed of a series of narrow lines separated by the fundamental accelerator frequency ( $3 \mathrm{GHz}$ in most cases). In certain conditions, a single line can be obtained [14] with a narrow bandwith of the order of $10^{-4}$. Due to these reasons and also to the success of the mid-infrared facilities, many FELs are now under development in FIR.

On storage ring based FELs, the laser operates very closed to the Fourier Limit close to perfect synchronism. When the FEL is detuned, bot the spectral and the temporal width widen.

Generally, the electron and optical pulses are longer than on a linac (10-100 p sec) but correlatively bandwidth as small as $10^{-4}$ to $10^{-6}$ can be made.

In the XUV and X ray spectral ranges, future SASE FELs may suffer from a rather large bandwith $(1 \%)$ due to the fact that the amplification would be starting from noise. This is likely to be improved by using part of the spontaneous emission, properly filtered by a monochromator, as a "seeder" for the SASE [6]. The short electron pulses envisioned (100 fs) would allow interesting real time studies, particularly in biology.

In addition, FELs using an optical resonator present a very good transverse coherence, because the optical mode builds from multi-turns in the optical cavity.

We will now summarize the FEL user facilities characteristics and applications.

\section{Far-infrared facilities}

As mentioned above, the first ever FEL facility was built specifically in FIR at Santa-Barbara by L. Elias [11] and started early after the initial success at Stanford. This facility uses a van de Graf $6 \mathrm{MeV}$ electrostatic accelerator. However, such a device can sustain a current of less than $1 \mathrm{~mA}$, although a current of a few Amps is needed for the FEL. The solution is to use a tandem of these machines, the second one decelerating the electrons after their interaction with the FEL and bringing them again to the potential of the gun of the first. This scheme has the advantage to lead to a very high efficiency of the FEL, if the recuperation rate is good enough $(\approx 99.9 \%)$ to allow quasi-cw operation. In practice, this has never been done yet, but there exist several FELs under development trying to take advantage of this possibility. For example, a microwave FEL is developed in Holland [15], in order to produce a cw MW beam aimed at fusion plasma heating. Due to imperfect recuperation, the pulse length of the Santa-Barbara FEL is approximately $10 \mu \mathrm{s}$.

The laser beam properties are governed by the pseudo-cw structure of the pulses: the peak laser power is of the order of a few kWatts. This power is sufficient for some pumpprobe experiment, although the pulse length is quite long. There is an effort being made in order to obtain picosecond long pulses by slicing it with a shutter: a mode-locked external laser focused on a intracavity silicon plate - in fact, the short pulses and the tens of MW range of power of linac based FEL seems to be better adapted to non-linear physics, which is one of the main interest of the FEL. The laser bandwidth of the electrostatic FEL is limited by the machine stability and energy drift due to imperfect recuperation to about $10^{-3}$, but can be made much smaller by sampling during the pulse. The laser is very wide band (60 to $800 \mu \mathrm{m})$, by having 3 different electron beam lines. The tunability is made by changing the energy which is somewhat more difficult than by changing the undulator gap (usually done on RF linacs). The applications deal mostly with semiconductors and quantum wells physics. A bibliography can be found in [16].

The Santa Barbara machine is the only far-infrared facility in the world. Lasing has been recently obtained with an electrostatic machine at Tel-Aviv University [17]. This machine has the peculiarity of being rather compact, the undulator being brought to the machine high potential 
(instead of the electron gun). A project exists at Orlando [18].

One RF linac based FEL is working the millimeter wavelength range at ENEA in Italy [19]. A few applications have been started. Interestingly, this laser has been a test bench for the physics of FIR pulsed FELs [14].

\section{Mid-infrared facilities}

Most of the FEL users facilities function in the mid-infrared range $(2$ to $200 \mu \mathrm{m})$ by using RF linac based machines. Their common characteristics are the followings:

- The time structure is made of a series of micro-pulses a few ps long. The train of micro-pulses is a few $\mu$ s long on room temperature linacs. On superconducting devices this train is several ms long. In order to produce high average powers, some laboratories are building high duty cycle superconducting accelerators [21] with beam recovery.

- As explained above, the FEL pulse length and linewidth are nearly transform limited and can be varied continuously over an order of magnitude by varying simply the optical cavity length. A small linewidth is used for nonlinear spectroscopy (Fig. 4), although a small pulse length is used for pump-probe and photon echo type of experiments.

- The peak power is of the order of 1 to $100 \mathrm{MW}$. The power averaged over the macropulses is a few $\mathrm{kW}$ and the average power is of the order of $1 \mathrm{~W}$. The average power can reach a few $\mathrm{kW}$ when the duty cycle is high, i.e. the macropulse lasts several $\mathrm{msec}$ at a repetition rate of several Hz: this is allowed with superconducting accelerators and such a high average power has been recently produced at Jefferson laboratory and at JAERI [21].

- Large tuning range ( 1 to 2 decades) and easy tunability, due to the large optical gain, are ordinarily obtained with those machines. The wavelength can be easily swept by controlling the undulator magnetic field ( $K$ parameter of formula 1 ). The beam is linarly polarized.

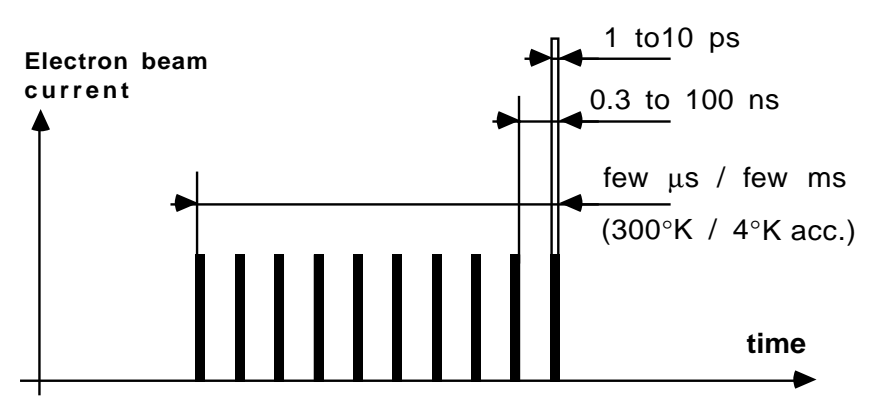

Figure 3. Time structure of pulsed RF linear accelerators: the spacing between the short micropulses has to be equal to the round trip time of light in the optical cavity (or to a divider).
Let us now briefly review these facilities worlwide. By "facility" we intend machines providing typically 1000 hours/year of laser beam to users. Therefore many FELs, existing or in projects, are not mentioned in this review.

The first RF linac FEL facility was implemented by J. Madey at Stanford [22] in the mid-80's. This machine uses a so-called "RF" gun, which is more compact than usual linac "injectors". However, in this configuration, the micropulse separation is as short as 300 ps, which limits the energy available in each pulse as well as the pump-probe studies capabilities. Also the spectral range $(2-8 \mu \mathrm{m})$ is presently rather limited for an FEL facility, particularly in the long wavelength range. Nevertheless this machine played a very important role as it demonstrated the reliability of the RF linac FEL and produced the first applications in the field [23]. It was later moved to Duke (USA).

After completion of his own machine, J. Madey started a company, "Sierra lasers", aimed at producing and selling clones of his FEL. A sole specimen was sold to Vanderbilt University, which precipitated the bankruptcy of the company. After several technical problems, the Vanderbilt FEL his now very active in medical studies [24], which are funded by a special program of the US government.

The superconducting linac with which the first FEL was made has been refurbished and is now operated as a user facility operating between 2 and $100 \mu \mathrm{m}$ [25]. Applications are made in various fields [26] such as Solid State and Surface Science, Molecular Materials, Chemistry, Medecine and Biophysics (http://www.stanford.edu/group/FEL/). Noticeably, interesting photon echos experiments have been performed by D. Fayer et al., particularly on biological materials [27].

A superconducting linac with beam recovery has produced an average power of several $\mathrm{kW}$ at Jefferson laboratory in Virginia (USA) at a wavelength of $3 \mu \mathrm{m}$. It is planned that this FEL becomes a user facility, particularly in the field

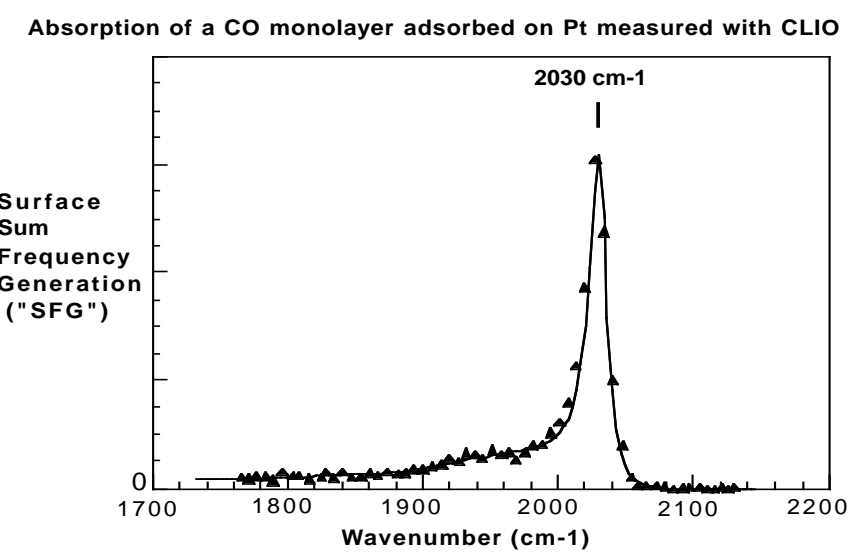

Figure 4. Example of non-linear spectroscopy performed with an FEL [20]. Such a spectrum is recorded in a few minutes by sweeping the undulator gap. 


\section{Original articles}

of material processing. The wavelength range will be extended toward the near-UV.

In Japan, the FELI laboratory involves several FELs covering from the UV to the mid-infrared and projected to cover also the FIR. The facility started recently and pursue several kinds of experiments [28]. The JAERI superconducting linac has recently produced a large average power at $20 \mu \mathrm{m}$ [21].

In Europe, there are two facilities: FELIX, in the Netherlands and CLIO, in France, which will be discussed in more details below. FELIX [29] uses two electron bends, which allow covering the range 5 to $200 \mu \mathrm{m}$. Most of FELIX applications are in the field of semiconductors and quantum wells [30], in collaration with UK groups. Also, Surface Science, Molecular and Atomic Physics have been studied $[31]^{2}$. These facilities started in 1992-93. EEC researchers have free access to them and support through a specific European program, after submission of their projects to an international program committee. Another facility (unpublished, except on the web) using a superconducting linac is being built in Dresden (RFA).

A large number of FEL experiments in the infrared, which are not used regularly for applications, exist [11].

\section{The CLIO facility}

The "CLIO" infrared free-electron laser at Orsay is a typical example of a user facility operating in the mid-infrared [32], offering more than 2000 hours/year to users. It is based on a linear accelerator working between 20 and $50 \mathrm{MeV}$. Its spectral range spans 3 to $60 \mu \mathrm{m}$. It will be extended to $100 \mu \mathrm{m}$ by modifying the machine in order to operate at lower energy (10 to $15 \mathrm{MeV}$ ), while keeping the high electron beam quality needed for an FEL.

The "two-colour" operation of the laser is a unique characteristic of CLIO allowing pump-probe experiments at different wavelengths (Fig. 6). The CLIO undulator is composed of two identical undulators, each $1 \mathrm{~m}$ long, independently adjustable. This combination has allowed us to demonstrate, up to $20 \mu \mathrm{m}$, the simultaneously lasing on two-colour [33] independently adjustable. By outcoupling the cavity with a hole in one mirror, a difference as large as $60 \%$ is produced. This allows new pump-probe applications as the two-colour are synchronized to within $1 \mathrm{ps}$.

There are 4 users stations where the beam can be commuted. Two of them are dedicated to particular experimental techniques:

- The first station is a diagnostic station and a "general purpose" user room.

- One station is dedicated to surface physics by Sum Frequency Generation (see below): it uses a Nd:YAG laser synchronized with CLIO. This experiment is entirely computerized and SFG scans comprising hundreds of

\footnotetext{
${ }^{2}$ More information can be found at: http://ns2.rijnh.n1/n4/f1234.htm
}

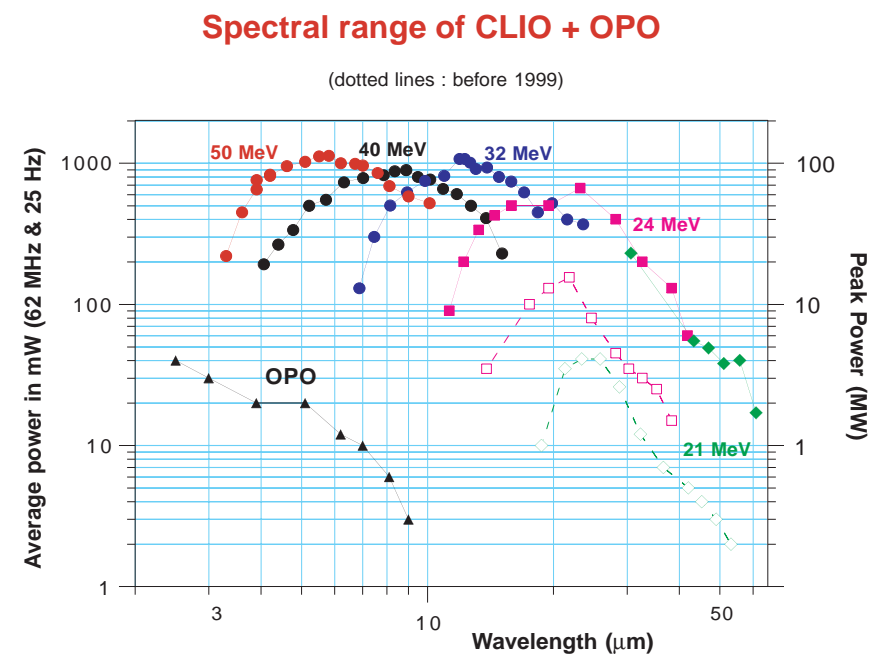

Figure 5. Spectral range and tunability at each energy of the CLIO FEL. At each accelerator energy, the wavelength can be swept continuously by modifying the undulator magnetic field.

averaged points (Fig. 4) can be recorded in minutes. An ultrahigh vacuum chamber is available for studies of contamination-free surfaces.

- The $3 \mathrm{~d}$ is a pump-probe and photon echo set-up dedicated now to the study of molecules in various environments and, in the near future, of biological molecules.

- A $4^{\text {th }}$ room will be equipped with a Nd:YILF laser pumping visible and UV OPOs synchronized with CLIO. It will allow to improve the SFG signal/noise ratio by an order of magnitude and to perform pump-probe experiment with a ps resolution.

The distribution of projects using CLIO since 1992 is the following:

- $30 \%$ for surface studies by SFG;

- $22 \%$ for electrochemistry studied by SFG;

- $22 \%$ for the study of molecules in matrices;

- $12 \%$ for semi-conductors and quantum wells;

- $7 \%$ for near field infrared microscopy;

- $3.5 \%$ for surface photoemission;

- $3.5 \%$ for medicine;

\section{Semiconductors \& quantum wells}

Semiconductors studies have been conducted by several groups [34]. Although the volume of this activity is modest on CLIO, they have been particularly successful: due to its large peak power and easy tunability, the FEL is particularly suited for non-linear studies in semiconductors and quantum wells. Kinetics in the ns and ps ranges range has been made. Pump-probe measurements on the ps time scales have permitted the observation of coherent transient grating effects of narrow gap semiconductors and the study of third-order nonlinearities. Another success is the study of the optical 


\section{Original articles}

\section{INTER-SUB-BAND STIMULATED EMISSION}
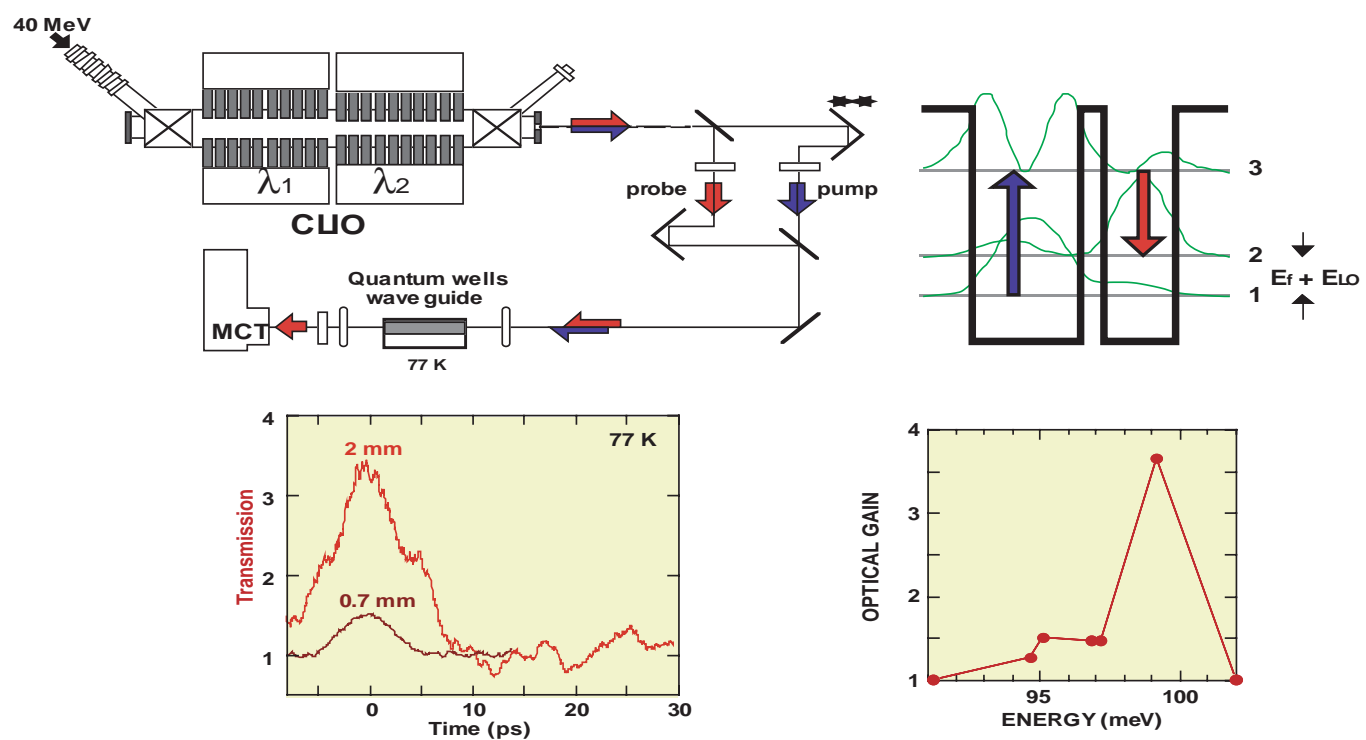

Figure 6. Scheme of the 2-colours free-electron laser applied to study of optical pumping in quantum wells by a pump-probe technique at 9.2 and $12.5 \mu \mathrm{m}$ respectively. The 2 undulators are set at 2 different magnetic gaps in order to produce these 2 wavelengths. The sample possesses a double well structure allowing stimulated emission. The first colour is used to populate the excited level of the first well, which relaxes into the second. This relaxation is stimulated by the second colour: The $2 \mathrm{~mm}$ long sample exhibits more transmission (i.e. gain) than the $0.7 \mathrm{~mm}$ one, which demonstrate stimulated emission.

pumping in quantum wells using the two-colour FEL for the pump and probe beams [35].

\section{Surface studies by SFG}

The Sum Frequency Generation ("SFG") on surfaces is a technique used to perform the spectroscopy of adsorbed species on surfaces. A (fixed wavelength) pulsed YAG laser is mixed with a (tunable) high power infrared laser: if the substrate is centrosymetric only the surface contributes to the process, which become resonant if the infrared wavelength corresponds to an absorption arising from the surface. Therefore the nonlinearity of the process, requiring high peak power, is efficient in discriminating the surface from the bulk. The FEL is particularly suited for this application since it is widely tunable and of high power. The SFG allows one to characterize the molecular structure and the coupling of the molecule to the substrate, surface or interface.

In practice, this technique is the more demanding user experiment in term of FEL intensity and wavelength stability, in order to obtain good signal to noise ratio spectra. It is therefore particularly important to monitor in real time the beam intensity, wavelength and linewidth, as explained above.

A typical result obtained in electrochemistry is shown in figure 7: SFG allows one to observe specifically the phenomena taking place at the surface of an electrode in a solvent $[20,36]$. Noticeable results have been obtained on the electrochemisty and surface science.

\section{Molecules in matrices}

Several research projects since 1992 deal with the subject of molecules in rare gas matrices. In particular, one has been conducted by P. Roubin et al. [37]. They have studied the kinetics of $\mathrm{D}_{2}$ ethane molecules isomerization in a matrix of Argon at a temperature of about $10 \mathrm{~K}$. CLIO has allowed them to study the efficiency of various absorption band located in the range of 4 to $12 \mu \mathrm{m}$. It opens a new class of experiments to study mode-selective chemistry that require high-powered, short-pulsed sources capable of exciting the full range of molecular vibrations. Projects are underway to study the relaxation and the dissociation of organic molecules in different matrices. Following the pioneering work made at Stanford, a new CLIO station is dedicated, either by pump-probe and by photon echos [38], to the study of the vibrationnal relaxation of molecules in various environment (solid, liquid, sol-gel) (Fig. 8).

\section{Near-field microscopy}

Near field microscopy has been developed for years in the visible part of the spectrum. One method is to employ a small optical fiber capturing part of an evanescent wave generated at a surface or interface. With a sufficiently small fiber, the Rayleigh criterion can be overcome. High 


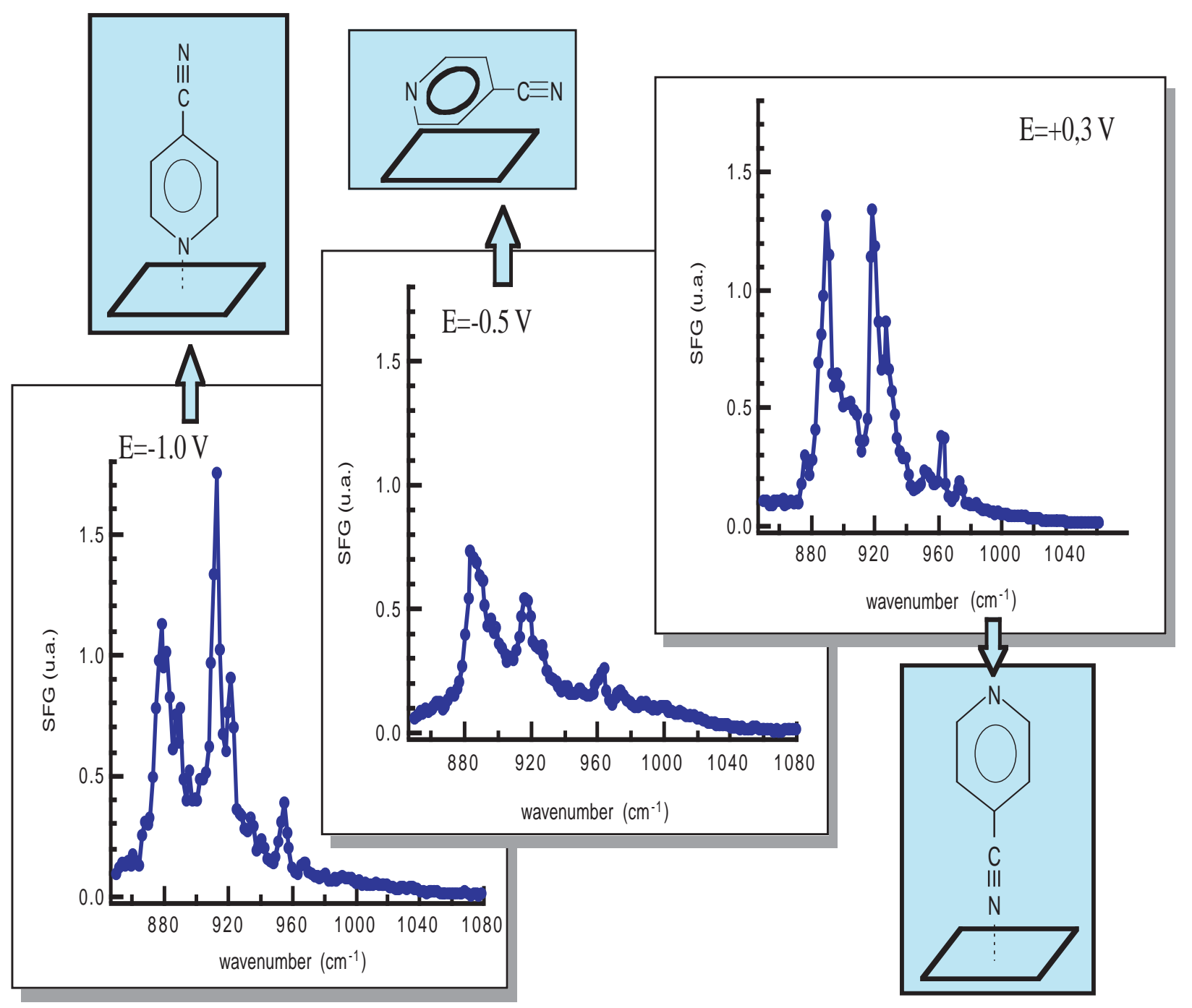

Figure 7. SFG (Sum Frequency Generation) spectra obtained with CLIO FEL: In situ study of the orientation of 4-cyanopyridine adsorbed on $\mathrm{Au}(111)$ as a function of the potential. At different electrochemical potentials, the molecules takes different orientations with respect to the surface. These orientations are probed by SFG, which is sensitive to the orientation of the dipoles. Solid lines: numerical fit for the various orientations of the molecule (O. Pluchery and A. Tadjeddine, Physica Status Solidi, vol. 175(1) 1999).

resolution surface spectromicroscopy can be undertaken by these means. The goal is to perform "chemical imaging" with a subwavelength lateral resolution. However, due to the lack of powerful infrared tunable lasers this had not been done until now in the infrared spectral range. At CLIO, a group coming from the St-Gobain-CNRS laboratory has undertaken such a project [39], which is now developed locally.

\section{Others}

Other studies have been made or are underway. In particular multiphoton induced electron emission has been observed on various metal surfaces, when irradiated at the level of only a few $\mathrm{MW} / \mathrm{cm}^{2}$. This effect is very surprising since, more than 40 photons being necessary to extract the electrons, such a process should have a very low probability [40]. Experiments in medicine (photoablation) have also been recently performed [41]. Probing the pseudo-gap of high Tc superconductors has been recently undertaken using the 2-colors capability of CLIO in the region $15-25 \mu \mathrm{m}$.

\section{Facilities in the ultra violet}

FELs in the UV are either based on Linear Accelerators or on storage rings (see Fig. 9) for which the FEL is naturally 

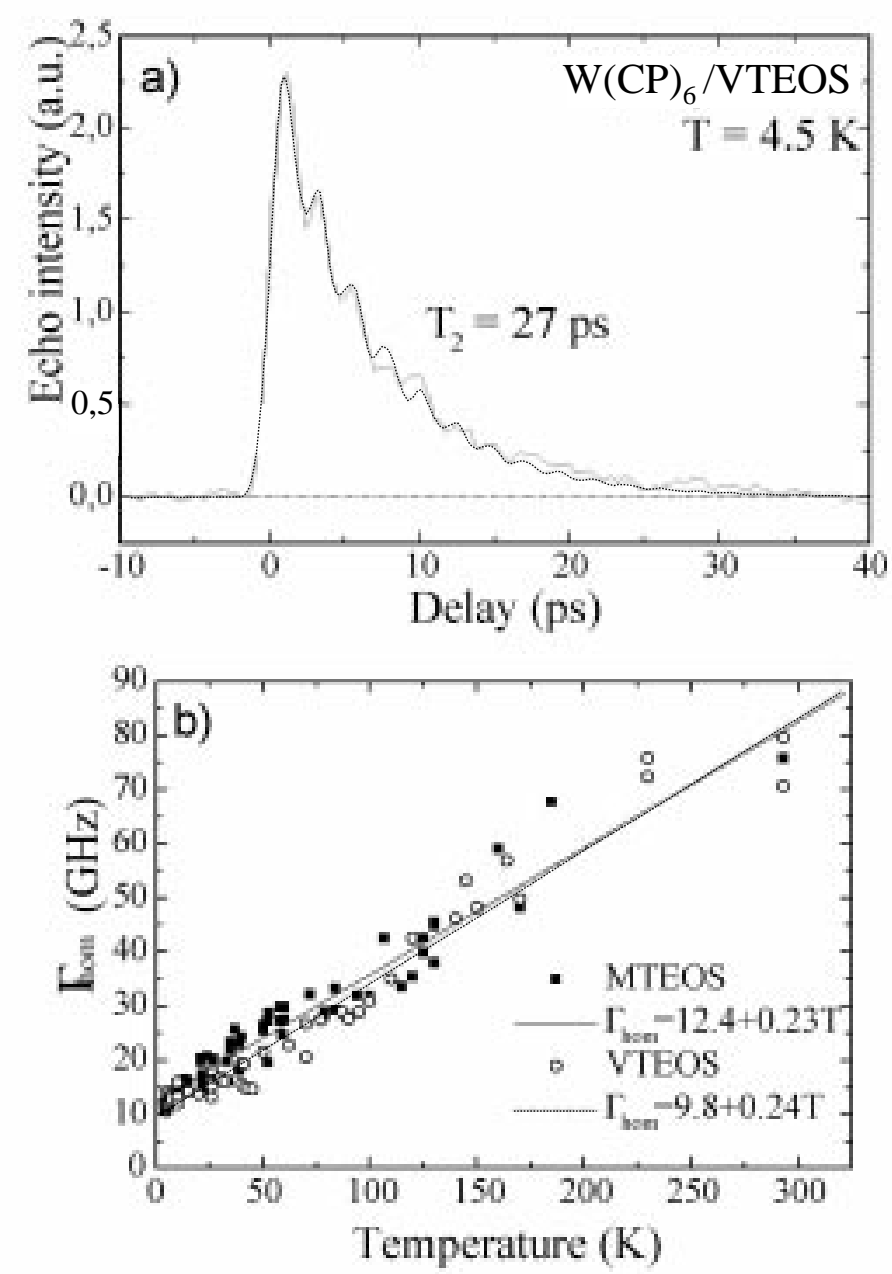

Figure 8. Photon echo measurements, from Galaup et al.[38] of the $\mathrm{CO}$ stretching mode in $\mathrm{W}(\mathrm{CO})_{6}$ in to different xerogels: methyltriethoxysilane (MTEOS) and vinyl-triethoxysilane (VTEOS). Upper curve: a typical echo signal showing the beating oscillations between the $(v=) 0 \rightarrow 1$ to $1 \rightarrow 2$ transitions (dotted line is a fit). Lower: temperature evolution of the homogeneous width $\left(=1 / . \mathrm{T}_{2}\right)$.

synchronised with synchrotron radiation. The scientific use of Storage Ring Free Electron Lasers in the UV started more recently, in 1993 on Super-ACO [42]. As compared to infrared FEL, the gain is lower and the optics are more critical, so 10 years separate the first lasing on a storage ring [43] in the visible and the first use of UV FELs.

Presently, the spectral range covers by UV FELs is shown in figure 10. There are six storage ring FELs in operation (Super-ACO [44], UVSOR [45], NIJI-4 [46], Duke [47], Delta [48], Elettra [49]) with a beam energy ranging between 0.8 to $1.5 \mathrm{GeV}$. The Duke FEL has already reached the VUV range. The average power of Storage ring FEL is proportional to the power emitted by synchrotron radiation, and increases significantly when relatively high energy beam

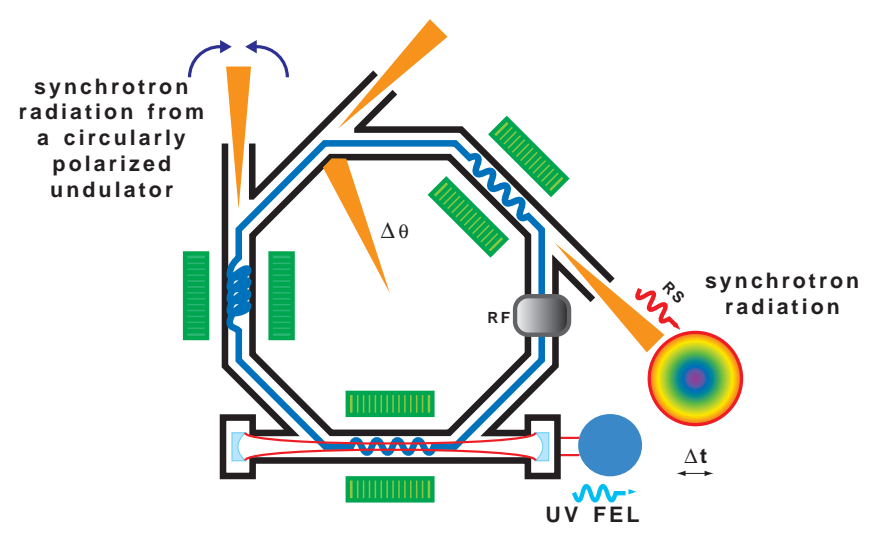

Figure 9. Scheme of a storage ring providing both the FEL and synchrotron radiation for pump-probe experiments.

are used. Besides, two UVFELs were achieved on LINAC, one on Los Alamos [50] presently shut down, and at FELI [51] in Japan.

UV LINAC driven FEL sources exhibit the same temporal structure, as in the infrared. SRFEL consists of a series of a few ps micropulses at a high repetition rate (several $\mathrm{MHz}$ ). Sometimes, an additional macrotemporal structure at the ms range can appear when the optical pulses bouncing in the optical resonator and the electron bunches stored in the ring are not perfectly synchronized. The relative spectral line is of the order of $0.01 \%$, allowing spectroscopic applications to be performed. With a Fabry-Perot etalon [52] in the optical resonator, the VEPP3 FEL demonstrated a relative linewidth of $10^{-6}$. Storage ring FELs operate very close to the Fourier limit.

The FEL is linearly polarized with planar undulator, and circular with helical undulators (as on UVSOR or Elettra).

So far, the Super-ACO FEL provide an average of 350 hours of beam-time for the users since 1994, and the Duke FEL offered 600 hours of operation for users in 1999, including 500 hours in the spontaneous emission mode. New user facitilies (buildings...) are there built this year. The European Elettra FEL is the first one implemented on a third generation synchrotron light source. The laser oscillation has been obtained one year and a half after the beginning of the project, and a user programme is ready to pioneering experiments.

The temporal structure of the FEL pulse is well adapted to time resolved fluorescence technique, as first demonstrated on the Super-ACO FEL [42].

A SRFEL is really a unique tool for performing twocolour experiments in various scientific domains, coupling both the laser source and synchrotron radiation (SR) [53], as shown in figure 9. Previous work was carried out on high frequency laser, mode-locked with synchrotron radiation [54]. Coupling an SRFEL with SR presents several 
advantages: natural synchronisation of the two pulsed sources, possibility of dynamical studies by scanning the SR to FEL delay, high repetition rate $(\mathrm{MHz})$ and pulse to pulse natural synchronisation, tunability of both sources (SR from the IR to the X-ray, and the FEL tunability being defined by the gain over losses ratio), polarization of both sources, photon flux and brilliance: the intensity delivered by an FEL is four order of magnitude greater than SR, already significantly more intense than conventional X-ray sources. Typical brilliance up to $10^{26} \mathrm{ph} / \mathrm{s} / \mathrm{mm}^{2} / \mathrm{mrad}^{2} / 0.1 \% \mathrm{BW}$ can be achieved with storage ring FEL sources.

\section{The Super-ACO UV FEL}

So far, the majority of the user experiments have been performed on Super-ACO. The motivation was more to demonstrate the possible uses on various scientific domains, than to extend the FEL radiation to the shortest wavelength as possible. Consequently, the FEL operation has been maintained at $800 \mathrm{MeV}$, the nominal energy of the ring, for the compatibility with the users of synchrotron radiation. A high current operation has also been established [55]. Moreover, the FEL issue has been dramatically improved, with a longitudinal feedback system allowing to reduce the FEL pulse jitters down to a small fraction of its width, the spectral drift to less than $10^{-5}$, and the intensity fluctuation to $1 \%$, during several hours without readjustment of the FEL itself [56].

The Super-ACO presently covers from the visible to $300 \mathrm{~nm}$, with $300 \mathrm{~mW}$ of average power at $350 \mathrm{~nm}$. The FEL pulse duration is usually 20 ps with a linewidth of $0.3 \AA$. The Super-ACO FEL is linearly polarized.

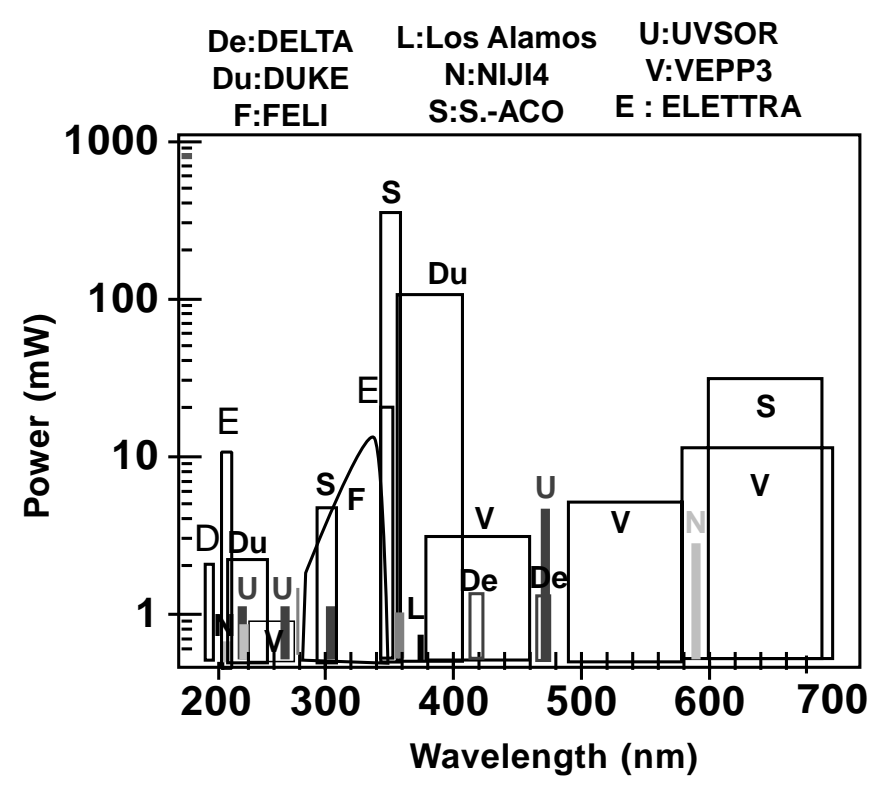

Figure 10. FEL sources in the UV
The FEL can presently be combined [53] with the normal synchrotron radiation beamlines of the facility, or with a new SA5 beamline covering from the UV to $20 \mu \mathrm{m}$. So far, the FEL transport and timing has been developed mainly for the SU3 photo-emission beamline on an undulator, and for the SA5 beamlines. In the future, experiments will be carried out also in combination with the adjustable polarization undulator beamline SU5 [57].

\section{Photochemistry and photobiology in solution}

The UV illumination of a solution in the the absorption bands of the molecule to a singulet electronic state can lead to various schemes of desexcitation, such as fluorescence, phophorescence after inter-system crossing, that can be studied with an UV FEL.

The feasibility of using the Super-ACO storage ring as a UV light source was demonstrated with the study of polarized fluorescence decays of the reduced nicotinamide adenine dinucleotide coenzyme (NADH) in aqueous solution, using the single photon counting (SPC) technique [42]. A complete fluorescence experiment (see Fig. 11) required about 30 min of data acquisition, during which the best integrated instrumental response limited by the detection had a FWHM of 110 ps with fluctuations of the absolute position of the apparatus function smaller than 5-10 ps, and a high number of counts $\left(10^{5}\right.$ at the maximum). The measurements performed led to the unambiguous separation of two close lifetime components of $0.28 \mathrm{~ns}$ and $0.62 \mathrm{~ns}$ in the fluorescence decays of $\mathrm{NADH}$ at $20^{\circ} \mathrm{C}$, in good agreement with previous works. The thermodynamic parameters obtained from temperature studies show that the NADH fluorescence heterogeneity is consistent with the ground state folding

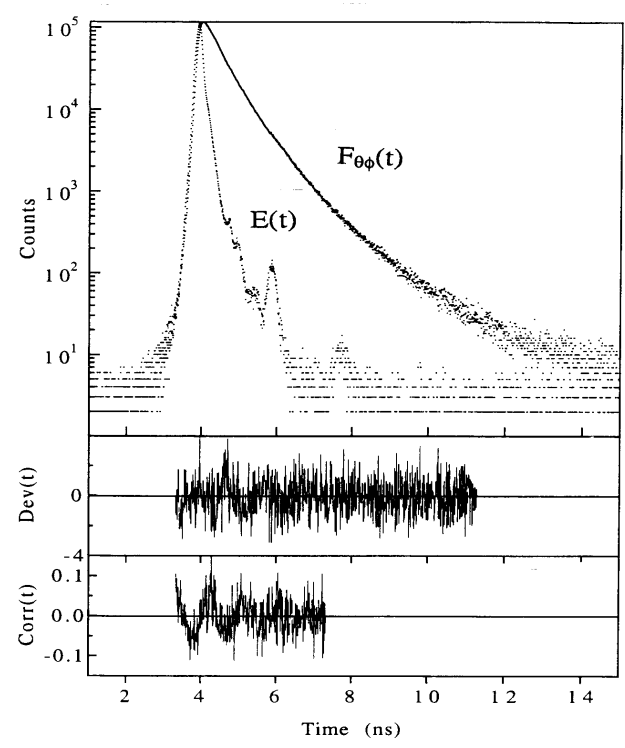

Figure 11. Fluorescence decay and instrumental function for a standrat acquisition. Standart deviation and Correlation function. 


\section{Original articles}

equilibrium of the coenzyme, as characterized by many other spectroscopic techniques. From the fluorescence anisotropy decays versus temperature, an apparent hydrodynamic radius of about $6 \AA$ is determined, while on the other hand, a large initial depolarization of the fluorescence indicates a fast independent motion of the nicotinamide ring. It is in good agreement with the volume of the folded configuration obtained by method of Van der Waals (see Fig. 12).

A transient absorption experiment where the FEL excites the solution, which is probed by the white synchrotron radiation has been recently developed [58]. Recent experiments showed a time resolved two-colour effect on a dye (POPOP) and on the acrinide. Various biological chromophore such as proteins, coenzymes and quinones, pigments and neurotransmettors, nucleic acides and basis could be analysed. Studies of intercalent drugs of DNA or photochimiotherapy and artifical nucleases are planned, using the Super-ACO FEL.

\section{Surface physics and condensed matter}

The first user applications combining the FEL with synchrotron radiation were performed in surface physics [59], for the study of the surface photovoltage effect (SPV). The SPV effect induced by the the Super-ACO FEL on a semiconductor interface has been probed by core level photoemission with synchrotron radiation from an undulator beamline. The SPV resulting from the creation of hole-electron pairs induced by the FEL, modifies the electronic band structure at the interface, and the core level photoemission peaks are displaced. The variation of the SPV signal versus the FEL to synchrotron radiation delay show the recombination processus at the interface. Studies were carried out on $\mathrm{Si}, \mathrm{GaAs} / \mathrm{Ag}, \mathrm{Si} / \mathrm{Au}, \mathrm{Si} / \mathrm{SiO}_{2}$. In this last case, the sign of the SPV signal depends on the thickness of the deposited silica.

First experiments are under way on two-color photo-emission.

Large gap semi-conductors such as photocarriers can be studied with an UV FEL excitation probed by infrared syn- chrotron radiation [60]. First result has also been obtained on kaolinite [60]. Identically, thin films and molecules adsorbed on surfaces could be studied.

Studies using the Photo Electron Emission Miscroscopy using both the spontaneous emission of the undulator and the FEL have been carried out at Duke University [61]. A microspcopy experiment is planned on the Elettra FEL [49]. First use of the gamma rays produced by Compton Scattering from the FEL has started at Duke University [62].

\section{UV and VUV FEL studies in gas phase}

Future UV FEL will provide average power in the 1-50 W range, together with VUV radiation by coherent harmonic generation. New types of experiments will then be possible, such as FEL induced photodissociation, analysis of exotic states such as ions and radicals, and non-linear applications [63].

\section{Conclusion}

Numerous infrared FEL facilities are now operating. Several UV FELs are starting applications programs. Original work using FEL facilities has been performed in various scientific domains and recognized by the international community (more than 250 published papers on the applications, not including the review or the FEL physic articles). Also, many studies made with FELs have resulted in international recognition (such as invitations of their authors in international conferences). The infrared FELs have proven to be a valuable source of radiation, with practically no competitors in the mid and far infrared spectral ranges. The UV SRFELs have a specific interest due to the coupling with the broadband synchrotron radiation. Shorter wavelengths FELs are studied experimentally, particularly at DESY (German synchrotron radiation laboratory in Hamburg, RFA).

\section{References}

1. Deacon, D.; Elias, L.; Madey, J.; Ramian, G.; Schwettman, H.; Smith, T. Phys. Rev. Lett. 1977, 38, 892.

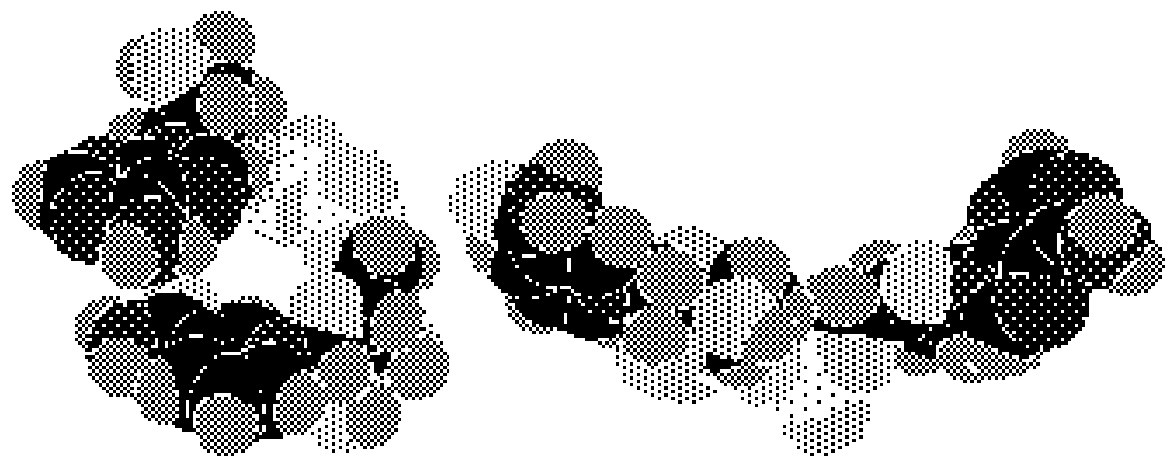

Figure 12. Conformations of NADH a) folded, b) unfolded 
2. Laser handbook; Amsterdam: North Holland, 1990, vol. 6; Brau, C. Free-electron lasers; San Diego, CA, USA: Academic Press, 1990.

3. Ortega, J. M.; Billardon, M.; Jezequel, G.; Thiry, P.; Petroff, Y. J. Phys. 1984, 45, 1883.

4. Kulipanov, G. et al. Nucl. Instr. \& Meth. 1990, A296, 3; Hama, H.; Kimura, K.; Hosaka, M.; Yamazaki, J.; Kinoshita, T. Tokyo: Feli Ionics publishing Co, AFEL97, P17-24; Yamazaki, T. et al. Nucl. Instr. Meth. 1993, A331, 27; Yamazaki, K. et al. Lasing below $300 \mathrm{~nm}$ in the NIJI-IV FEL, Proced. 20th FEL conf, Williamsburg, August 1998; Litvinenko, V. et al. Nucl. Inst. meth A 2000, in press; Nölle, D. private communication; Nölle, D. et al. Proceedings Free Electron laser Conf, August 1996, Roma; Amsterdam: Noth Holland, 1997, II3; Couprie, M. E.; Garzella, D.; Billardon, M. Europhysics Lett. 1993, 21(9), 909-914; Couprie, M. E. et al. Nucl. Inst. Meth. 1999, in press.

5. Prazeres, R. et al. Europhysics Letters 1987, 4, 817.

6. Bonifacio, B. et al. Opt. Commun. 1984, 50, 373; Murphy, J.; Pellegrini, C. Nucl. Instr. and Meth. 1985, A237, 159; Kim, K.-J. Phys. Rev. Lett. 1986, 57, 1871; Tatchyn, R. et al. Nucl. Instr. and Meth. 1996, A375, 274; Rossbach, J. Nucl. Instr. and Meth. 1996, A375, 269.

7. Prazeres, R. et al. Phys. Rev. Lett. 1997, 78, 2124; Hogan, M. et al. Phys. Rev. Lett., 1998, 80; Andruskow, J. et al. Phys. Rev. Lett., 2000.

8. Glotin, F. et al. Phys. Rev. Lett. 1996, 77, 3130; Handbook of synchrotron radiation; Amsterdam: North-Holland publishing, Vol.1 to 4; Nutarelli, D.; Couprie, M. E.; Nahon, L.; Bakker, R.; Delboulbé, A.; Roux, R.; Visentin, B.; Billardon, M. Nucl. Instr. Meth. A 1998, 407, 459-466; Litvinenko, V. N. et al. Phys. Rev. Lett. 1997, 78(24), 4569- 4572; Hosaka, M.; Hama, H.; Kimura, K.; Yamazaki, J.; Kinoshita, T. Free Elecron Laser and its Appl. in Asia; Tokyo: Ionics Publish. Co., pp 25-30; Ohgaki, H.; Yamada, K.; Sei, N.; Yamazaki, T.; Noguchi, T.; Mikado, T.; Sugiyama, S. Proceedings of the FEL96 conference; Amsterdam: North Holland, 1997, II-14.

9. Walker, R. P. et al. Proceed. 20th Intern. FEL conference, Williamsburg, August 1998; Couprie, M. E.; Nutarelli, D.; Billardon, M. Nucl. Inst. Meth. B 1998, 144, 66-74.

10. Nguyen, et al., Nucl. Instr. \& Meth. 1995, A358, 3; Schmerge, J. et al. Nucl. Instr. \& Meth. 1995, A358, 24.

11. Proceedings of FEL conferences; Nucl. Instr. \& Meth. 1985, A237; 1986, A250; 1987, A259; 1988, A272; 1989, A285; 1990, A296; 1991, A304; 1992, A318; 1993, A331; 1994, $A 341 ; 1995, A 358 ; 1996, A 375 ; 1997, A 393 ; 1998, A 407$; 1999, A429.

12. Elias, L. Phys. Rev. Lett. 1979, 16, 977; Elias, L.; Hu, J.; Ramian, G. Nucl. Instr. \& Meth. 1985, A237, 203.

13. Hooper, B. et al. Nucl. Instr. and Meth. 1988, A272, 96; Glotin, F.; Chaput, R.; Jaroszynski, D.; Prazeres, R.; Ortega, J.M. Phys. Rev. Lett. 1993, 71, 2587; Knippels, G.; Mols, R.; Van der Meer, A.; Oepts, D.; Van Amersfoort, P. Phys. Rev. Lett. 1995, 75, 1755.

14. Oepts, D. et al. Phys. Rev. Lett. 1992, 68, 3543; Ciocci, et al. Phys. Rev. Lett. 1993, 70, 928.

15. Urbanus, W. et al. Nucl. Instr. \& Meth. 1998, A407, 327.

16. Allen, S. et al. Nucl. Instr. \& Meth. 1995, A358, 536; Craig, K. et al. Phys. Rev. Lett. 1996, 76, 2382; Unterrainer, K. et al. Phys. Rev. Lett. 1996, 70, 2973.

17. Cohen, M. et al. Nucl. Instr. \& Meth. 1994, A341, ABS57.

18. Elias, L. et al. Nucl. Instr. \& Meth. 1991, A304, 219.
19. Ciocci, F. et al. Phys. Rev. Lett. 1991, 66, 699.

20. Peremans, A. et al. J. of Electr. Spectr. \& Relat. Phenom. 1993, 64/65, 391.

21. Neil, G. et al. Phys. Rev. Letter 2000, 84(4), 662-665; Minehara, E.J.; Suwamura, M.; Nagai, R.; Kikuzawa, N.; Nishimori, N.; Sugimoto, M. Nucl. Inst. Meth. 1999, A429, 911; Hajima et al. Proc. 22 th Intern. Conf Duke Univ., August 2000.

22. Benson, S.; Madey, J.; Schultz, J.; Marc, M.; Wadensweiler, W.; Westenkow, G. Nucl. Instr. \& Meth. 1986, A250, 39.

23. Benson, S.; Madey, J.; Straight, R.; Hooper, B. Laser Appl. 1989, 1, 49; Fann, W. et al. Phys. Rev. Lett. 1989, 62, 1492.

24. Edwards, G. et al. Lett. to Nature 1994, 371, 416; Edwards, G. Optical Engineering 1995, 34, 1524.

25. Schwettman, H.; Smith, T.; Swent, R. Nucl. Instr. \& Meth. 1996, A375, 662.

26. Xu, Z. et al. Phys. Rev. 1995, B51, 10631; Engholm, J. et al. Phys. Rev. Lett. 1996, 77, 1302.

27. Dlott, D.; Fayer, M. IEEE J. of Quant. El. 1991, 27, 2697; Rella, C. et al. J. Phys. Chem. 1996, 100, 15620.

28. Saeki, K. et al. Nucl. Instr. \& Meth. 1996, A375, 10.

29. Bakker, R. et al. IEEE J. of Quant. El. 1994, 30, 1635; Oepts, D. et al. Infrared Phys. Technology 1995, 36, 297.

30. Murdin, B. et al. Semic. Sci. Technol. 1994, 9, 1554; Murdin, B. et al. Phys. Rev. 1997, B55, 5171.

31. van der Ham, E.; Vrehen, Q.; Eliel, E. Optics Lett. 1996, 21, 1448; Putter, M.; Helden, G.; Meijer, G. Chem. Phys. Lett. 1996, 258, 118; Walker, R. et al. J. Biomed. Optics 1997, accepted.

32. Ortega, J.-M. et al. Nucl. Instr. and Methods 1996, A375, 618.

33. Jaroszynski, D.; Prazeres, R.; Glotin, F.; Ortega, J.M. Phys. Rev. Lett., 1994, 72, 2387.

34. Murdin, B. et al. Optical and Quantum Electronics 1993, 25, 171; Murdin, B.N. et al. Optical Materials 1993, 2, 89-93; Hughes, S.; Ciesla, C.; Murdin, B.; Pidgeon, C.; Jaroszynski, D.; Prazeres, R. J. Appl. Phys. 1995, 78, Duboz, J.Y. et al. J. Appl. Phys. 1995, 77, 6492; J. Appl. Phys. 1995, 78, 1224; Boucaud, P.; Julien, F. H.; Prazeres, R.; Ortega, J.M., Sagnes, I.; Campidelli, Y. Appl. Phys. Lett. 1996, 69, 3069; Sauvage, S. et al. Appl. Phys. Lett. 1998, 73, 3818.

35. Gauthier-Lafaye, O. et al. Appl. Phys. Lett. 1997, 70, 3197; Brunhes, T. et al. Appl. Phys. Lett., 1999, 75, 835; Brunhes, T. et al. Phys. Rev. B 2000, 61, 5561.

36. Peremans, A. et al. Nuclear Instr. \& Meth. 1994, A341, 146; Peremans, A.; Tadjeddine, A. Phys. Rev. Lett. 1994, 73, 3010; Tadjeddine, A.; Peremans, A.; Guyot-Sionnest, P. Chem. Phys. Lett. 1995, 247, 243; Tadjeddine, A.; Peremans, A.; GuyotSionnest, P. Surface Science 1995, 335, 210; Peremans, A.; Tadjeddine, A. J. Chem. Phys. 1995, 103(16), 7197; Tadjeddine, A.; Peremans, A. Journal de Chimie Physique 1996, 93, 662; Le Rille, A. et al. Chem. Phys. Lett. 1997, 271, 95; Hébert, P. et al. J. Electroanal. Chem. 1998, 447, 5-9; Tadjeddine, A.; Le Rille, A. J. of Electroanalytical Chemistry 1999, 467, 238.

37. Roubin, P.; Cardonato, S.; Dubost, H.; Lefèvre, J.; Ortega, J. M. J. Chem. Phys. 1994, 101 (4), 3403; Roubin, P. et al., J. Chem. Phys. 1997, 107, 7800.

38. Broquier, M. et al. Opt. Comm. 1995, 118, 255; Galaup, M. et al. J. of luminescence 2000, 86, 363; Crépin, C. et al., Laser Chem. 1999, 19, 65; Crépin, C. et al. Phys. Rev. Lett. 2000, $85,964$. 


\section{Original articles}

39. Piednoir, A.; Creuzet, F.; Licoppe, C.: Ortega, J. M. Ultramicroscopy 1995, 57, 282; Piednoir, A.; Creuzet, F.; Licoppe, C. Opt. Comm. 1996, 129, 414; Gross, N. et al., to be published.

40. Farkas, G. et al. J. Phys. B. 1998, 31, L461-L468.

41. Ostertag, M.; Schmiedt, K.C.; Walker, R.; Ortega, J. M.; Bende, M.; Jean, B. Investigative Ophth. \& Vis. Sci. 1996, 37, 3, S571; Glotin, F. et al., SPIE to be published.

42. Couprie, M. E.; Tauc, P.; Merola, F.; Delboulbé, A.; Garzella, D.; Hara, T.; Billardon, M. Rev. of Scient. Instrum. 1994, 65(5), 1485-1495.

43. Billardon, M.; Elleaume, P.; Ortega, J. M.; Bazin, C.; Bergher, M.; Velghe, M.; Deacon, D. A. G.; Petroff, Y. Phys. Rev. Lett. 1983, $51,1652$.

44. Couprie, M. E.; Hara, T.; Gontier, D.; Troussel, P.; Garzella, D.; Delboulbé, A.; Billardon, M. Phys. Rev. E 1996, 53, n²2, 1871-1889; Couprie, M. E.; Garzella, D.; Delboulbé, A.; Velghe, M.; Billardon, M. Nucl. Instrum. Meth. 1993, A331, 37-41; Roux, R.; Couprie, M. E.; Bakker, R. J.; Garzella, D.; Nutarelli, D.; Nahon, L.; Billardon, M. Phys. Rev. E. 1998, 58 , n ${ }^{\circ}$, 6584-6593; Garzella, D. et al. Proced. FEL Conf, Hamburg, August 1999.

45. Hama, H. Nucl. Instrum. Meth. 1996, A375, 57-61; Kimura, K. et al. Nucl. Instrum. Meth. 1996, A375, 62-66.

46. Yamazaki, T.; Sugiyama, S.; Ohgaki, H.; Sei, N.; Mikado, T.; Noguchi, T.; Chiwaki, M.; Suzuki, R.; Kawai, M.; Yokoyama, M.; Owaki, K.; Hamada, S.; Aizawa, K.; Oku, Y.; Iwata, A.; Yoshiwa, M. Nucl. Instr. Meth. 1993, A331, 27; Yamazaki, K. et al. Nucl. Inst. Meth. 1999, A429, 159-164.

47. Litvinenko, V. N.; Burnham, B.; Madey, J. M. J.; Park, S. H.; Wu, Y. Nucl. Instrum. Meth. 1996, A375, 46-52; Livinenko, V. et al. Nucl. Inst. Meth. 1999, A429, 151-158; Proceed. 21th FEL conf. Hamburg, 1999.

48. Nölle, D. Proceed. FEL conf, Hamburg, 1999.

49. Walker, R. et al. Nucl. Inst. Meth. 1999, A429, 179-184; Walker, R. et al. The European UV/VUV FEL project at Elettra, EPAC 2000, Vienna, Walker R. et al. Proceed. 22th International Free Electron Laser Conference, Duke Univ, August. 2000.

50. O'Shea, P.G.; Bender, S.C.; Byrd, D.A.; Early, J.W.; Feldman, D.W.; Fortgang, C.M.; Goldstein, J. C.; Newnam, B. E.; Sheffield, R. L.; Warren, R.W.; Zaugg, T. J. Nucl. Inst. Meth. 1994, A341, 7-11.

51. T. Tomimasu et al. Free Elecron Laser and its appl. in Asia; Tokyo: Ionics Publish. Co., 1997, pp 65-98.

52. Couprie, M. E.; Gavrilov, N.G.; Kulipanov, G.N.; Litvinienko, V.N.; Pinaev, I.V.; Popik, V. M.; Skrinsky, A. N.; Vinokurov, N. A. NIM 1991, A304, 47-52.

53. Couprie, M. E. Nucl. Inst. Meth. 1997, A393, 13-17; Hara, T.; Couprie, M. E.; Garzella, D.; Nahon, L.; Marsi, M.; Bakker, R.; Billardon, M. VUV 11, Tokyo; J. Electron Spectroscopy \& Related Phenomena 1996, 80, 317-320.
54. Mitani et al. Rev. Scient. Instr. 1989, 60, 1569; Ederer et al. NIM 1992, A319, 250; Lacoursière, J.; Meyer, M.; Nahon, L.; Morin, P.; Larzillière, M. Nucl. Instrum. Methods Phys. Res. 1994, A351, 545.

55. Roux, R.; Couprie, M. E.; Bakker, R. J.; Garzella, D.; Nutarelli, D.; Nahon, L.; Billardon, M. Phys. Rev. E. 1998, 58, $\mathrm{n}^{\circ} 5,6584-6593$.

56. Couprie, M. E.; Garzella, D.; Hara, T.; Codarbox, J. H.; Billardon, M. Nucl. Inst. Meth. A 1995, 358, 374.

57. Nahon, L.; Corlier, M.; Peaupardin, P.; Marteau, F.; Marcouillé, O.; Alacaraz, C. J. Synch. Rad. 1998, 5, 428-430; Ito, K.; Lagarde, B.; Polack, F.; Alcaraz, C.; Nahon, L. J. Synch. Rad. 1998, 5, 839-841.

58. Renault, E.; Nahon, L.; Nutarelli, D.; Garzella, D.; Couprie, M. E.; Mérola, F. Proceedings SPIE, Biomedical applications of Free-Electrons Lasers, Edwards, G. S.; Sutherland, J. C. Editors, Vol. 3925, 2000, pp 29-39; Mérola, F.; Fontaine, M.P.; Renault, E.; Polewski, K.; Couprie, M. E.; Garzella, D.; Nahon, L.; Billardon, M. Proceed. 12th International Congress on Photobiology, Vienna (Austria) 1-6th sept. 1996; Landmarks in photobiology, Hönigsmann, H.; Knobler, R. M.; Trautinger, F.; Jori, G. Editors, OEMF, 1998, pp 193-199.

59. Marsi, M.; Couprie, M. E.; Nahon, L.; Garzella, D.; Delboulbé, A.; Hara, T.; Bakker, R.; Indlekofer, G.; Billardon, M.; Taleb-Ibrahimi, A. Appl. Phys. Lett. 1997, 70(7), 895-897; Marsi, M.; Couprie, M. E.; Nahon, L.; Garzella, D.; Bakker, R.; Delboulbé, A.; Nutarelli, D.; Roux, R.; Visentin, B.; Grupp, C.; Indlekofer, G.; Panaccione, G.; Taleb-Ibrahimi, A.; Billardon, M. Nucl. Inst. Meth. 1997, A393, 548-551; Marsi, M.; Nahon, L.; Couprie, M. E.; Garzella, D.; Hara, T.; Bakker, R.; Billardon, M.; Delboulbé, A.; Indlekofer, G.; TalebIbrahimi, A. Journal of Electron Spectroscopy and Related Phenomena 1998, 94, 149-157; Marsi, M.; Belkhou, R.; Grupp, C.; Panaccione, G.; Taleb-Ibrahimi, A.; Nahon, L.; Garzella, D.; Nutarelli, D.; Renault, E.; Roux, R.; Couprie, M.E.; Billardon, M. Phys. Rev. B 2000, 61, R5070-R5073.

60. Carr, G. L. et al. Semicond. Sci. Technol. 1993, 8, 922-92; Nahon, L.; Renault, E. Couprie, M-E.; Nutarelli, D.; Garzella, D.; Billardon, M.; Carr, G. L.; Williams, G. P.; Dumas, P. Proceedings SPIE, 1999, Vol. 3775, pp 145-154; International Symposium on Optical Sciences: accelerator-based sources of Infra-Red and spectroscopic applications II, Denver (USA), 18-23juillet 1999.

61. Nemanich, R. Proceed 22th Intern. FEL Conf and User Workshop, Duke Univ, August 2000, pp 8-24.

62. Weller, H. R. Proceed 22th Intern. FEL Conf. and User Workshop, Duke Univ, August 2000, pp 8-24.

63. Le laser à Électrons Libres UV-VUV sur l'anneau SOLEIL : performances réactualisées et Argumentation Scientifique, mai 1997, Rapport Soleil. 